\title{
What is inflammatory bowel disease?
}

\author{
LAURINGTON R DACOSTA, MD(LOND), FRCP(LOND), FACP, FRCPC
}

T HE TERM INFLAMMATORY BOWEL disease (IBD) refers to two inflammatory disorders of unknown etiology ulcerative colitis and Crohn's disease.

In the majority of cases the clinical manifestations, anatomic distribution and pathological features make distinction of the two disorders easy. However, when the disease is restricted to the colon, overlap in the clinical and histological manifestations of the disorders makes distinction impossible in up to 10 to $15 \%$ of cases. Are the two disorders manifestation of the same etiopathogenic process, or are they distinct diseases, with clinical and pathological manifestations overlapping because of the bowel's limited repertoire of responses to damage?

\section{ULCERATIVE COLITIS}

Ulcerative colitis, first described in 1875 by Wilks and Moxon (1), is characterized clinically by recurring episodes of bloody diarrhea and pathologically by diffuse inflammatory changes confined to the mucosa, and to a lesser extent to the adjacent submucosa, of the rectum and left colon in continuity. In about $30 \%$ of cases the entire colon is involved. The clinical manifestations vary depending on the extent of bowel involved and the severity of the inflammatory response. In approximately $50 \%$ of cases the clinical attack is classified as mild, $30 \%$ as moderate and $20 \%$ as severe.

\section{CROHN'S DISEASE}

In 1913 Dalziel (2) first described a number of patients with what was probably Crohn's disease. Crohn, Ginsburg and Oppenheimer in 1932 (3) described the clinical features of what they called terminal ileitis. It soon became obvious that the disease was not confined to the terminal ileum and the name was changed to regional ileitis. In 1960, Lockhart-Mummery et al (4) described granulomatous colitis affecting the colon. The name of the disease was then changed to Crohn's disease.

Like ulcerative colitis, Crohn's disease is a chronic inflammatory disorder of unknown etiology. In contrast to ulcerative colitis the characteristic inflammatory response is transmural with granulomas occurring in 30 to $60 \%$ of cases. The disease has a predilection for the terminal ileum but may affect any part of the gastrointestinal tract including the mouth and perianal region. The inflammatory process is usually discontinuous with diseased segments of

Division of Gastroenterology, Queens University, Kingston, Ontario

Correspondence: Dr LR DaCosta, Division of Gastroenterology, Queen's University, 166 Brock Street, Kingston, Ontario K7L 5G2. Telephone (416) 544-0499

bowel separated by healthy segments (skip lesion). Small bowel or severe perianal involvement easily distinguishes Crohn's disease from ulcerative colitis. However, when the disease is confined to the colon, differentiation between the two diseases may be impossible even after colectomy. These cases are referred to as 'indeterminate' colitis and have overlapping clinical and pathological features of both diseases. Many of the indeterminate cases were described in pathological specimens obtained at emergency surgery for acute, severe disease rather than specimens obtained at elective surgery. Perhaps severe disease blurs the distinguishing characteristics of the two disorders. Nonetheless, milder forms of indeterminate colitis are seen in nonresected specimens.

\section{DISTINGUISHING FEATURES}

There are a number of features that have proven useful in distinguishing ulcerative colitis from Crohn's disease. Rectal sparing on sigmoidoscopy occurs in $50 \%$ of Crohn's disease but is virtually never seen in ulcerative colitis. Skip lesions on either double contrast barium enema or colonoscopy occurs in Crohn's disease but not in ulcerative colitis. In ulcerative colitis the inflammatory response is diffuse and invariably involves the rectum and colon in continuity. The mucosa is friable, 
granular and hyperemic. In Crohn's disease the mucosa may be thickened and cobblestoned with aphthous ulcers or deep round, linear or serpiginous ulcers. The lesions are often separated by relatively normal-appearing mucosa.

Rectal biopsy may be helpful in differentiating the two disorders. The finding of discrete granulomas on colonic biopsy is highly suggestive of Crohn's disease. Unfortunately, this is seen in only 30 to $60 \%$ of biopsy specimens. In the absence of granulomas there are still histological features which are of value in distinguishing ulcerative colitis from Crohn's disease. In ulcerative colitis the inflammation is confined to the mucosa and, to a lesser extent, to the adjacent submucosa. There is marked depletion of goblet cell mucin, increased crypt distortion and frequent crypt abscesses. Patchy inflammation with preservation of normal crypt architecture and predominantly submucosal inflammation favours a diagnosis of Crohn's disease.

\section{EPIDEMIOLOGY}

There is some similarity in the epidemiology of ulcerative colitis and Crohn's disease. Both disorders are worldwide in their distribution. The diseases occur most frequently in the developed countries of northern Europe and North America, with intermediate frequency in Central Europe, the Middle East and Australia, and least frequently in Asia and Africa. The annual incidence of IBD worldwide is from five to 15 new cases per 100,000 population in countries where it has been studied.

The ratio of ulcerative colitis to Crohn's disease varies from region to region but in most countries ulcerative colitis appears to occur more frequently than Crohn's disease. Since its first description in 1875 , the incidence of ulcerative colitis has increased with each decade until the past three decades when it appears to have levelled off, with an annual incidence of four to 15 new cases per 100,000 population and a prevalence of 44 to 117 cases per 100,000 population.

It seems difficult to believe that clinicians of the early 20th century were unaware of the clinical manifestations and pathological findings of Crohn's disease. Since Crohn and his colleagues (3) described the disease in 1932, the incidence of the disease has increased significantly greater than can be explained by better diagnostic facilities and by differentiation of the disorder from ulcerative colitis and tuberculosis. In some countries, such as Sweden, the incidence of Crohn's disease now exceeds that of ulcerative colitis. The annual incidence of Crohn's disease varies from a low of 0.3 per 100,000 in Finland to a high of 13.5 per 100,000 in Minnesota. The prevalence varies from 28 to 108 cases per 100,000 population.

Epidemiological data on IBD in Canada is scanty. Mendelhoff (5) quotes the incidence of Crohn's disease in Sherbrooke, Quebec, as 0.7 cases per 100,000 population and the prevalence as 6.3 cases per 100,000 . These figures are low compared with published data for other countries. Recently, Pinchbeck et al (6) described the incidence of Crohn's disease and ulcerative colitis in Alberta as 10 and six cases per 100,000 population, respectively. The prevalence was 44 cases per 100,000 for Crohn's disease and 37.5 per 100,000 for ulcerative colitis.

Davis et al (7) estimated the incidence and prevalence rates of IBD in southern Alberta. The incidence of ulcerative colitis in females was 1.8 per 100,000 and in males 2.4 per 100,000 , while the incidence of Crohn's disease in females was 6.5 per 100,000 and in males 3.1 per 100,000 . The prevalence rate for ulcerative colitis was 22.4 cases per 100,000 population in females and 24.7 per 100,000 in males. The prevalence rate for Crohn's disease was 63.7 per 100,000 for females and 38.1 per 100,000 for males. Depew et al (8), reporting on the clinical presentation of Crohn's disease in southeastern Ontario, described a prevalence of 33.1 cases per 100,000 population. More studies are obviously needed to document accurately the incidence of IBD in Canada.

The ratio of males to females is approximately equal, although women appear to have a $30 \%$ increased risk of developing IBD when population groups are predominantly English or northern European. The three most recent studies from Canada suggest that there is a slightly increased incidence in the female population.

The peak incidence of both diseases is between the ages of 15 and 25 years. Both diseases appear to be rare below the age of six and the incidence of ulcerative colitis appears to exceed that of Crohn's disease in the first decade. In some (but not all) series, there is an increase in incidence between ages 55 and 60 years.

IBD is more common in whites than in nonwhites. Both diseases are more common in Jews, particularly in studies from the United States and Scandinavia. Studies in Israel suggest that the incidence in Jews born in the western hemisphere is higher than in Jews born in Israel.

Approximately $18 \%$ of patients with IBD have family members with either ulcerative colitis or Crohn's disease, compared with $4 \%$ of controls. The tendency to have a relative with IBD is greater if the proband has Crohn's disease. This familial aggregation of IBD is probably genetic rather than the result of a common environmental factor; however, to date no genetic markers have been found to support a genetic basis for IBD.

\section{POSSIBLE ETIOLOGY OF IBD}

Environmental factors are probably important in the etiology of IBD; however, no convincing reproducible evidence has been found to propel into the limelight any one item as the etiological agent. Several studies suggest that there is an increased risk of ulcerative colitis in nonsmokers and in former heavy smokers. The data for Crohn's disease indicate that individuals who smoke are at greater risk of developing that disease. An intriguing association has also been seen between IBD and the use of oral contraceptives. Some studies show a significant increase in the incidence of ulcerative colitis and Crohn's disease in oral contraceptive users whereas other studies have supported an association between oral contraception and Crohn's disease but not ulcerative colitis. It seems that the relative risk for oral contraceptive users developing either 
ulcerative colitis or Crohn's disease returns towards normal when the oral contraception is discontinued. Mechanisms of this association are unknown.

A number of dietary items, such as milk, formula feeding, fibre and refined sugar, have been investigated as possible environmental factors. The results of the studies have been conflicting. No single item can be convincingly shown to play a significant etiological role.

The etiopathogenesis of IBD remains as elusive today as it did 60 years ago, despite intensive research efforts over the ensuing time. Infectious, genetic, allergic, psychogenic and immunological theories have been postulated over the years, but none is supported by adequate or convincing evidence. The two most popular theories remain the infectious and the immunological theories.

To date, no bacteria, fungus or virus has been consistently isolated from patients with ulcerative colitis. Transmission studies have been unsuccessful. Microbiological studies have also failed to reveal a specific etiological agent for Crohn's disease. Bacteria-free filtrates of Crohn's disease tissue were reported to produce granulomatous reactions in the footpads of mice and in the intestines of rabbits. Furthermore, these lesions could be transmitted from animal to animal. Other workers were unable to duplicate these findings and the transmissible agent was never identified. Viral studies have either been unrewarding or have yielded a variety of RNA or DNA viruses, none of which has been convincingly associated with Crohn's disease. Histological features of Crohn's disease suggest that a mycobacterium might be involved. Chiodini and colleagues (9) suggested an etiological relationship between Crohn's disease and a mycobacterium resembling paratuberculosis, which was isolated from a number of their patients. However, the mycobacterium has only been isolated from a relatively small number of patients and attempts at isolation and transmission have not been consistently successful.

Despite the failure to fulfil Koch's postulate for a causative agent, bacteria may play a significant role in the etiopathogenesis of IBD. Intestinal bac- teria contain peptides and cell wall polymers with potent inflammatory and immunoregulatory properties. These products are capable of producing acute intestinal inflammatory changes in experimental animals. These inflammatory agents may pass through the bowel wall in patients with IBD because of increased intestinal permeability. While not causative, bacteria and their products may amplify and perpetuate the intestinal damage.

The histological features of IBD, including infiltration with plasma cells, lymphocytes, macrophages and acute inflammatory cells, and the absence of any obvious infectious agent, led to the suggestion that abnormal immune responses were directed either against gut-associated antigens such as the normal bacteria flora or against the intestinal mucosa. A number of immunological abnormalities occurs in both ulcerative colitis and Crohn's disease patients. These abnormalities include: high titres of antibodies to colonic epithelial cells; antibodies to cross-reacting antibacterial antigens; cytotoxicity of circulating lymphocytes for colonic epithelial cells; abnormalities of immunoglobulin and complement patterns; decreased response of circulating lymphocytes to mitogenic stimulation; variable degrees of anergy to tuberculin and other antigens; and circulating antigen-antibody complexes. It is probable that these $\mathrm{ab}$ normal immune responses are the result of the disease process and do not represent etiopathogenic factors; however, these issues remain unresolved and the subject of ongoing research.

The role of inflammatory mediators in the etiopathogenesis of IBD has generated recent interest. The tissue responses seen in IBD, such as infiltration with inflammatory cells, vasodilation, and plasma exudation, might be the result of locally synthesized chemical mediators. The metabolites of arachidonic acid are potent chemical mediators. Increased tissue levels of prostaglandins have been demonstrated in acute experimental colitis and in IBD patients. Initially it was thought that inhibition of prostaglandins by sulphasalazine and 5-aminosalicylic acid (5-ASA) was the method of action of these drugs. However, treatment with potent inhibitors of prostaglandin synthesis, such as indomethacin, afforded no beneficial effect in IBD patients. Furthermore, sulphasalazine and 5 . ASA, effective drugs in the therapy of IBD, have very weak and inconsistent inhibitory action on prostaglandin synthesis. These findings suggest that prostaglandins play no significant role in the mucosal inflammatory response in IBD. Recent studies have shown that IBD patients synthesize a number of other metabolites of arachidonic acid in increased amounts. These include the potent chemotactic agent leukotriene B4. The synthesis of leukotrienes is inhibited by sulphasalazine and 5-ASA. The importance of these inflammatory mediators remains to be clarified.

IBD was once considered a psychosomatic disorder. The evidence supporting this theory was derived from retrospective and anecdotal reporting of the frequent occurrence of repressed hostility and dependency in IBD patients as well as an association between relapses of the disease and emotional traumatic events. More controlled studies have challenged these views. There now appears to be no higher incidence of psychiatric abnormalities in IBD patients than in the control population. Emotional traumatic events preceding an attack of ulcerative colitis occur in fewer than $20 \%$ of patients. As is true in any chronic debilitating disease, psychological and social factors are often prominent and require treatment.

Truelove (10) described antibodies to milk in patients with ulcerative colitis and suggested that these may have an etiological role. These have subsequently been shown to be no more common in ulcerative colitis patients than in the general population. Furthermore, there is no correlation between levels of milk antibodies and severity or relapses of ulcerative colitis.

It is tempting to speculate that polygenic inheritance provides the background in an individual in whom IBD may develop. Bacterial, viral or some as yet unidentified environmental factor(s) might be the initiating agent which sets up an abnormal immune response in the gastrointestinal tract. 
Bacterial peptides, cell wall polymers and inflammatory mediators all play a role in the tissue response. Increased intestinal permeability allows the entrance of various noxious substances into the mucosa and results in many of the abnormal immune responses identified in IBD. The initiating process results in the spectrum of pathological manifestations in the gastrointestinal tract. Those that develop transmural granulomatous lesions, that is Crohn's disease, also tend to have small bowel involvement, whereas those that develop a mucosal disease alone have ulcerative colitis. Unfortunately, there is no evidence to support the above unifying hypothesis.

\section{PATHOGENESIS OF IBD}

Ulcerative colitis may occur abruptly in a small percentage of patients but more usually it starts insidiously. The disease may present as proctitis, proctosigmoiditis or a pan proctocolitis. In ulcerative proctitis the inflammation is confined to the rectum. The patients present with intermittent rectal bleeding. They usually have normal bowel habits or even constipation and do not have any systemic manifestations of the disease. In proctosigmoiditis the inflammation spreads proximally in continuity from the rectum to the sigmoid and left colon. Common symptoms include diarrhea, with the passage of blood or mucus, lassitude and fever; systemic symptoms may occur but are rare. In pan proctocolitis the entire colon is involved in continuity. The symptoms include the passage of fragmented loose stools, blood and mucus intimately mixed with the stools, rectal urgency, tenesmus and systemic symptoms such as anorexia, malaise, fever, weight loss and tachycardia.

Crohn's disease also is strikingly variable in its severity and manifestations. In $20 \%$ of cases the onset is abrupt, in the rest the onset is insidious. Most patients have recurrent symptomatic episodes with gradual progression of the disease. In some the course is rapidly progressive and in others the disease may become apparent only with manifestation of one of the late complications. The clinical manifestations de- pend on the site and the extent of bowel involvement. The disease has a predilection for the terminal ileum.

The typical patient is a young adult with persistent, though not severe, diarrhea accompanied by fever, right lower quadrant abdominal pain, anorexia, nausea and vomiting. Examination reveals tenderness in the right lower quadrant and perhaps a palpable sausage-shaped mass of matted loops of bowel. The symptoms may persist for a few weeks and subside spontaneously only to recur again in a few weeks or months. Other modes of presentation include pain resembling acute appendicitis, recurrent periumbilical and right lower quadrant pain, obstructive in character and with associated nausea and vomiting. With more extensive small bowel involvement there may be steatorrhea with weight loss, anemia and other signs of malabsorption.

Crohn's colitis occurs in approximately $30 \%$ of patients. The clinical presentation is often insidious with nonbloody diarrhea, abdominal pain, fever, weight loss and anemia. Although rectal bleeding is not as common as in ulcerative colitis, it occurs in about $40 \%$ of patients with Crohn's disease of the colon. Anorectal disease is extremely common, occurring in $80 \%$ of patients, and consists of ulcers with or without fissures or superficial and deep fistulas. The ulceration and fistulization may erode and destroy the internal and external sphincters.

\section{COMPLICATIONS}

A number of complications occur in patients with IBD. Some of these are similar for both ulcerative colitis and Crohn's disease. The complications may be divided into local or intestinal complications, and extraintestinal or systemic complications.

The most important local or intestinal complication is the development of cancer. The risk of developing cancer in ulcerative colitis is dependent on the extent of the disease involvement of the bowel and the duration of the disease. In patients with total colonic involvement the risk of developing cancer in the first eight to 10 years is not significantly greater than that of the general population. After 10 years the risk increases by 0.5 to $1 \%$ per year and is accumulative. This risk is significant and the present policy is to advise surveillance colonoscopy with multiple biopsies from suspicious lesions and from every 5 to $10 \mathrm{~cm}$ of the normal bowel looking for dysplastic lesions. Surveillance colonoscopy should start at eight to 10 years. The incidence of cancer is also increased in Crohn's disease although to a lesser extent than in ulcerative colitis. The role of surveillance colonoscopy in this setting is as yet undetermined.

Other local or intestinal complications include stricture formation and acute or chronic obstruction. This occurs commonly in small bowel Crohn's disease but may also occur in Crohn's colitis. Benign strictures of the colon may occur in up to $10 \%$ of patients with ulcerative colitis. In these cases it is important to exclude malignancy. In Crohn's disease, fistulas may occur between areas of diseased bowel and normal bowel (enteroenteric or enterocolic), and between bowel and bladder (enterovesical), vagina (enterovaginal) or skin (enterocutaneous).

The extraintestinal complications have been classified into three main categories. The first group are primarily colitis-related and include manifestations involving the joints, skin, eyes and mouth. The second group are small bowel related and include malabsorption, nephrolithiasis, and cholelithiasis. They occur almost exclusively in Crohn's disease. The third group is nonspecific and includes hepatobiliary complications, amyloidosis and hydronephrosis.

Arthritis is the most common extraintestinal manifestation of IBD and occurs in up to $25 \%$ of patients. A migratory asymmetrical, seronegative, peripheral arthritis called 'colitic' arthritis is observed in 15 to $20 \%$ of IBD patients. It affects the knees, hips, ankles, wrists and elbows in decreasing frequency. Attacks of colitic arthritis tend to occur when the IBD is active.

Sacroileitis occurs in up to $10 \%$ of IBD patients. Ankylosing spondylitis is seen in $6 \%$ of patients, and occurs with equal frequency in patients with ulcera- 
tive colitis and Crohn's disease. Ninety per cent of patients with IBD and ankylosing spondylitis are associated with HLA B27 antigen. Unlike colitic arthritis, ankylosing spondylitis and sacroileitis may antedate the bowel symptoms and progress independently of the activity of the IBD.

Clubbing of the fingers occurs more commonly in Crohn's disease than in ulcerative colitis. Eye complications occur in up to $10 \%$ of patients, and include episcleritis, uveitis, iritis and cataracts. There is an association between the iritis, arthritis and disease activity in Crohn's disease patients.

Skin and mucus membrane involvement occurs in up to $10 \%$ of IBD patients and include erythema nodosum, pyoderma gangrenosum ( 1 to $4 \%$ ) and aphthous ulcerations of the mouth in Crohn's disease (less frequently in ulcerative colitis).

Hepatobiliary complications occur with varying reported frequency in IBD, depending on the source of the data. Many of the complications are asymptomatic with minimal or no biochemical abnormalities. Fatty liver and pericholangitis are the most common lesions identified on liver biopsy. With the advent of endoscopic retrograde cholangiopancreatography (ERCP) a significant number of patients with pericholangitis have been found to have sclerosing cholangitis. Most of the cases of sclerosing cholangitis occur in ulcerative colitis and appear to be an unusual finding in Crohn's disease. Nevertheless, increasing numbers of cases are being described in Crohn's disease patients. Other hepatobiliary lesions include chronic active hepatitis, granulomas, bile duct carcinoma, amyloidosis, and occlusion of the portal and hepatic veins.

Cholelithiasis occurs in up to $15 \%$ of patients with Crohn's disease of the terminal ileum or in patients who have had an ileal resection.

Nephrolithiasis due to oxalate stones occurs in patients with Crohn's disease of the terminal ileum or following resection of the terminal ileum.

Hematological abnormalities are common in IBD patients. Iron deficiency anemia due to blood loss is the most common type of anemia seen. Macrocytic anemia may occur due to folate deficiency as a result of sulphasalazine therapy or to impaired $\mathrm{B}_{12}$ absorption in Crohn's disease of the terminal ileum. Anemia of chronic disease occurs in both ulcerative colitis and Crohn's disease.

Growth retardation with delayed sexual maturation is a serious complication of Crohn's disease and, less frequently, ulcerative colitis. Prompt treatment of the IBD and appropriate nutritional replacement are imperative to prevent this disastrous complication from occurring.

\section{DIFFERENTIAL DIAGNOSIS OF IBD}

The diagnosis of IBD is based on a good history and physical examination, sigmoidoscopic appearances of the bowel, rectal biopsy, bacteriology and radiology. Disorders from which IBD must be differentiated include the specific colitides such as infectious colitis, antibiotic-associated colitis, radiation colitis, ischemic colitis, carcinoma of the colon, toxic or traumatic colonic injury, uremia and, on occasion, irritable bowel syndrome. The importance of establishing the correct diagnosis in each and every case cannot be overemphasized.

One of the disorders from which IBD must be distinguished is infectious colitis. In most instances infectious colitis is a self-limited disease lasting less than one week and requiring only supportive treatment in the form of fluid replacement. There is no need for specific agents as used in IBD, nor are there any long term implications. Bacterial causes of infectious colitis, such as salmonella, shigella, campylobacter, yersinia and Escherichia coli, can be confirmed in $48 \mathrm{~h}$ by stool cultures. With parasitic infections such as amoebiasis or giardiasis, microscopy of the stool - or of duodenal aspirate in the case of giardiasis - is important.

Sigmoidoscopy will confirm acute colitis but will not distinguish between IBD and infectious colitis. An acute inflammatory infiltrate with cryptitis may be seen in both IBD and infectious colitis; however, more chronic changes; such as crypt distortion, regeneration and atrophy, are usually absent in infectious colitis. Pseudomembranous colitis secondary to Clostridium difficile and associated with recent antibiotic therapy may mimic severe ulcerative colitis and require assay of the stool for $\mathrm{C}$ difficile cytotoxin. The finding on sigmoidoscopy of typical pseudomembranes confirmed by histology would help to distinguish pseudomembranous colitis from IBD.

Acute self-limited colitis also occurs in homosexuals. Here the infecting agents not only include those seen in the heterosexual population but also an increased prevalence of Neisseria gonorrhoea, Treponema palidum, Chlamydia trachomatous, herpes genitalia and cytomegalovirus. These infections may occur singly or in combination, and give rise to symptoms and sigmoidoscopic evidence of colitis or proctocolitis which must be distinguished from IBD. Immunocompromised patients and patients with the acquired immunodeficiency syndrome (AIDS) may have acute or chronic diarrhea due to a number of opportunistic infections including parasitic infections such as cryptosporidium, Isospora belli, Giardia lamblia and Entamoeba hystolitica; bacterial infections such as salmonella, shigella, yersinia and campylobacter; and viruses such as cytomegalovirus and herpes simplex.

IBD is usually readily distinguished from radiation colitis by the history of previous radiation therapy and the sigmoidoscopic and histological findings on rectal biopsy. Similarly, IBD is easily differentiated from carcinoma of the colon.

Differentiation from ischemic colitis, particularly in the older age group, is sometimes a challenge. In the classic case of acute abdominal pain followed by bloody diarrhea with typical radiological findings of thumb printing and the colonoscopic findings of mucosal edema and purpura, differentiation from ulcerative colitis or Crohn's disease is relatively easy. However, in the subacute or more chronic case of ischemic colitis the differentiation from either ulcerative colitis or, more often, Crohn's disease is indeed difficult. The 
disease may be patchy in nature and is often associated with chronic, deep ulcers resembling Crohn's disease. Histologically the findings may be similar to Crohn's disease; however, the finding of hemosiderin-laden macrophages is suggestive of ischemic colitis.

Usually there is little difficulty in distinguishing IBD from irritable bowel syndrome. In the latter condition the patient complains of abdominal pain associated with alternating constipation and diarrhea. Symptoms are rarely nocturnal, are not associated with systemic symptoms such as fever, weight loss or extraintestinal manifestations of IBD, and usually there is no blood in the

\section{REFERENCES}

1. Wilks S, Moxon W. Lectures on Pathological Anatomy, 2nd edn. London: Churchill, 1875:408, 672.

2. Dalziel TK. Chronic intestinal enteritis. Br Med J 1913;ii:1068-70.

3. Crohn BB, et al. Regional ileitis: A pathological and clinical entity. JAMA 1932;99:1323.

4. Lockhart-Mummery HE, Morson BC. Crohn's disease (regional enteritis) of the large intestine and its distinction from ulcerative colitis. Gut 1960;1:87.

5. Mendelhoff AI, Calkin BM. The epidemiology of inflammatory bowel stool unless the patient also has concomitant hemorrhoids. Sigmoidoscopic findings are usually normal as is rectal biopsy and barium enema. Manning (11) suggested that six symptoms were more common in irritable bowel syndrome than in organic disease such as IBD. These included: pain relieved by bowel motion; loose stools with onset of pain; increased bowel motions with the onset of pain; abdominal distention; mucus per rectum; and feeling of incomplete rectal emptying. Thompson et al (12) confirmed the reliability of these criteria in patients with IBD and irritable bowel syndrome.

In summary, IBD comprises two dis-

disease. In: Kirsner JB, Shorter RG, eds. Inflammatory Bowel Disease, 3rd edn. Philadelphia: Lea and Febriger, 1988:3-34.

6. Pinchbeck ER, et al. Inflammatory bowel disease in northern Alberta. J Clin Gastroenterol 1988;10:505-15.

7. David FG, et al. Inflammatory bowel disease, incidence and prevalence in southern Alberta. Can J Gastroenterol 1990;4:187-92.

8. Depew WT, et al. Clinical presentation and course of Crohn's disease in southeastern Ontario. Can J Gastroenterol 1988;2:107-16. orders: ulcerative colitis and Crohn's disease. Both are chronic debilitating inflammatory disorders affecting predominantly young people. There are many similarities between the two disorders including their epidemiology, etiopathogenesis and extraintestinal manifestations. However, there are certain distinct differences particularly when the small bowel is involved. Much work has gone into trying to find the cause for the diseases; however, despite a great deal of information which has been garnered, to date no single etiological agent has been identified. As a result there is no cure for IBD other than surgery in the case of ulcerative colitis.

9. Chiodini RJ, et al. Possible role of mycobacteria in inflammatory bowel disease I. An unclassified mycobacterium species isolated from patients with Crohn's disease. Dig Dis Sci 1973;29:1984.

10. Truelove SC. Ulcerative colitis provoked by milk. Br Med J 1961;i:154-60.

11. Manning AP, et al. Towards positive diagnosis of the irritable bowel. $\mathrm{Br}$ Med J 1978;ii:653.

12. Thompson WG, et al. Irritable bowel syndrome: Guidelines for diagnosis. Gastroenterol Int 1989:292-6. 


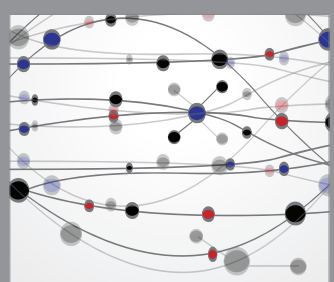

The Scientific World Journal
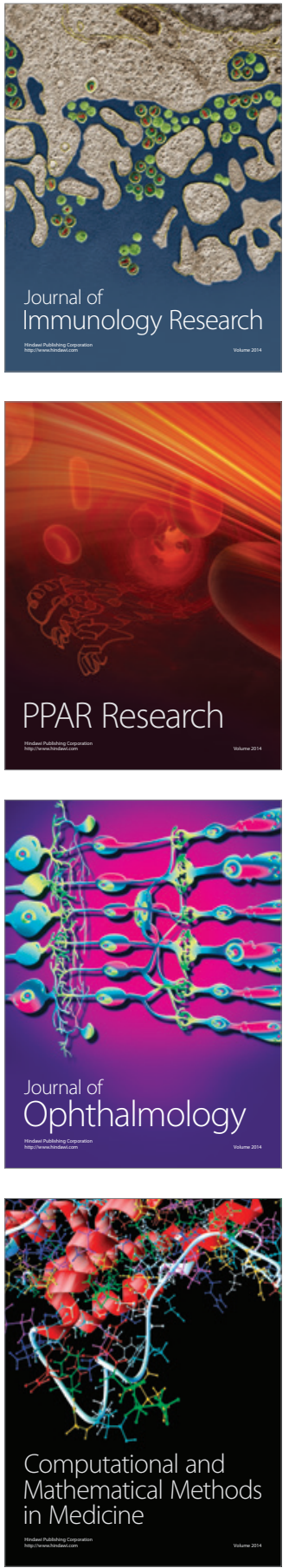

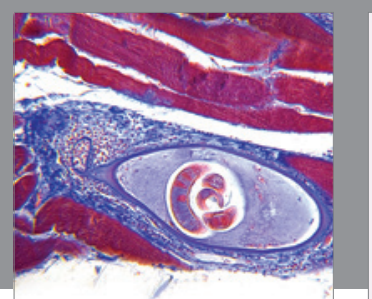

Gastroenterology Research and Practice

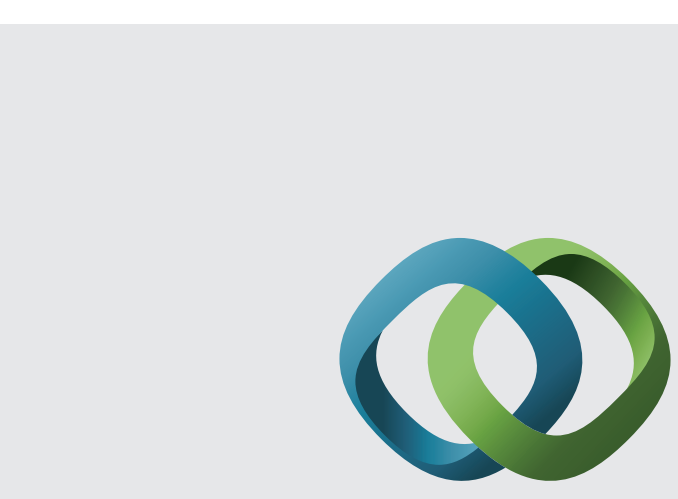

\section{Hindawi}

Submit your manuscripts at

http://www.hindawi.com
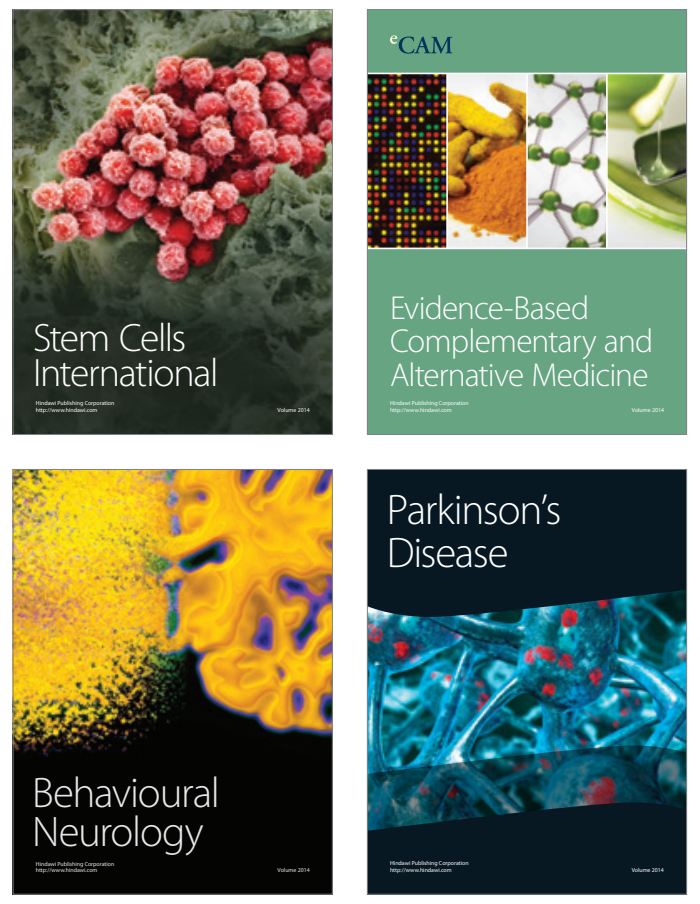
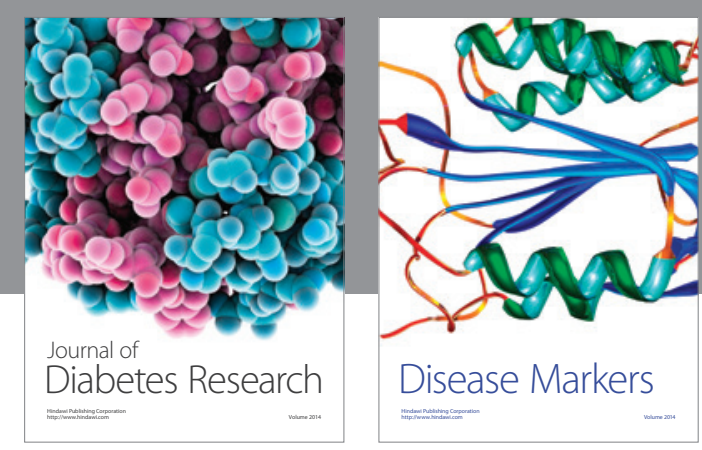

Disease Markers
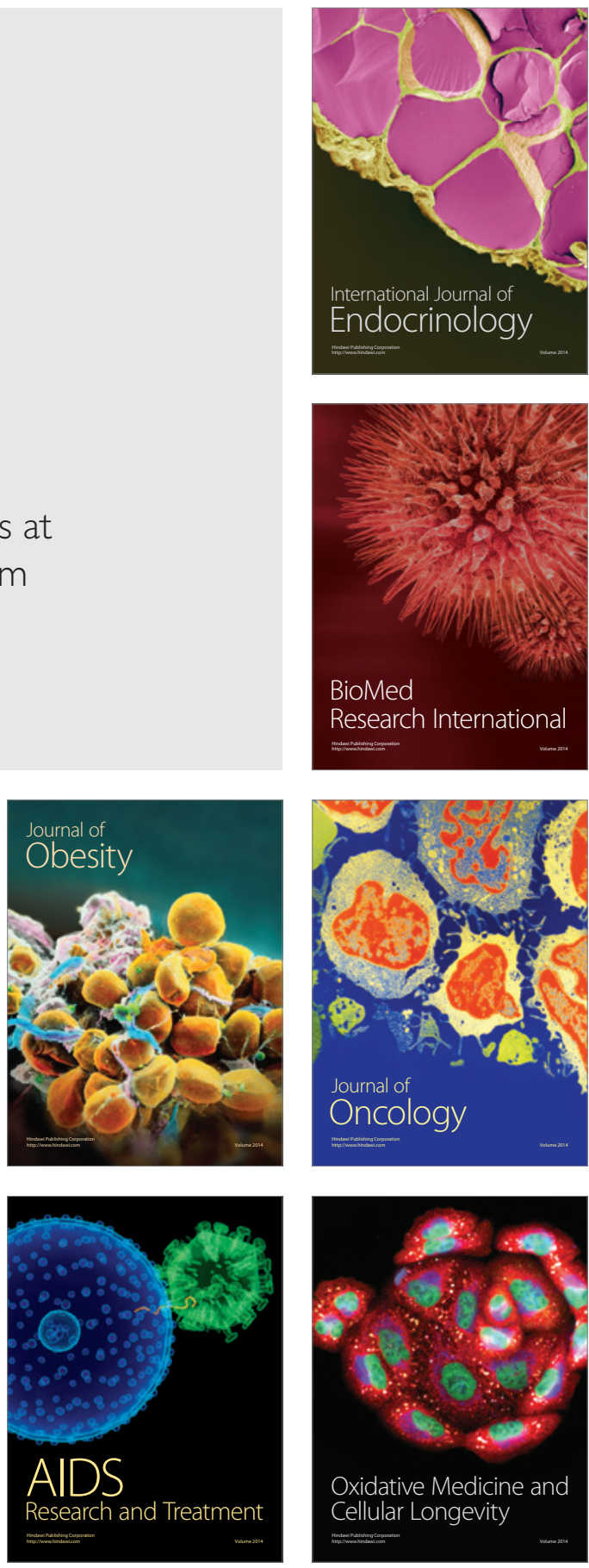